\title{
Fever-induced Brugada syndrome in a 9-year-old boy presenting with acute chest pain
}

\author{
Gülser Esen Besli ${ }^{1}$, Sema Yıldırım², İbrahim Akalın³ ${ }^{3}$ Yusuf İzzet Ayhan ${ }^{4}$, Merve Kısıoğlu², \\ Afig Berdeli ${ }^{5}$ \\ Departments of ${ }^{1}$ Pediatric Emergency, ${ }^{2}$ Pediatrics, ${ }^{3}$ Medical Genetics and ${ }^{4}$ Pediatric Cardiology, Istanbul Medeniyet \\ University Goztepe Training and Research Hospital, İstanbul, ${ }^{5}$ Department of Pediatrics, Molecular Medicine Laboratory, \\ Ege University Faculty of Medicine, İzmir, Turkey. E-mail: besliesen@gmail.com \\ Received: 1st March 2018, Accepted: 3rd May 2018
}

SUMMARY: Besli GE, Yıldırım S, Akalın I, Ayhan Yİ, Kısığlu M, Berdeli A. Fever-induced Brugada syndrome in a 9-year-old boy presenting with acute chest pain. Turk J Pediatr 2018; 60: 571-575.

Brugada syndrome, an arrhythmogenic disease, occurs due to mutations involving cardiac sodium channels. It is characterized by persistent or transient ST-segment elevation in the right precordial electrocardiogram leads that could be unmasked by several circumstances, with fever particularly. Molecular and cellular mechanisms leading to Brugada syndrome have not been completely elucidated. Mutations of the SCN5A gene encoding the pore-forming $\alpha$-subunit of the cardiac sodium channel protein have been attributed in the molecular diagnosis. Although this syndrome is well-known in adults, it is less frequently reported in infants and children. We describe a 9-year-old Turkish boy with a family history of sudden cardiac death, who presented with chest pain and fever-induced expression of the Brugada syndrome phenotype that might be associated with a mutation in SCN5A gene.

Key words: Brugada syndrome, cardiac sodium channel, chest pain, children, fever.

Brugada syndrome $(\mathrm{BrS})$ is a rare arrhythmogenic disease with a high risk of life-threatening ventricular dysrhythmias and sudden cardiac death (SCD) with no underlying structural heart disease. ${ }^{1}$ It is characterized by a typical pattern of ST-segment elevation in the right precordial leads on an electrocardiogram (ECG). ${ }^{1,2}$ This syndrome is a channelopathy leading to loss of function of the voltage-gated cardiac sodium channel. ${ }^{3,4}$ Although this syndrome is wellknown in adults, it is less frequently reported in infants and children. ${ }^{5}$ There is limited information about the prevalence, clinical presentation, and prognosis of $\mathrm{BrS}$ in pediatric patients. ${ }^{6}$ It is probably underdiagnosed due to dynamic and variable changes on ECGs and the clinical spectrum that can range from asymptomatic to SCD at first presentation. $3,6,7$ ECG patterns of BrS can be revealed by fever, drugs such as sodium channel blockers, vagotonic agents, tricyclic antidepressants, and electrolyte abnormalities. ${ }^{4-6,8,9}$ Fever was found to be the most common precipitating factor for ventricular dysrhythmias in a pediatric case series with BrS. ${ }^{6}$
Although the molecular and cellular mechanisms leading to $\mathrm{BrS}$ have not been completely elucidated, mutations of the SCN $5 \mathrm{~A}$ gene encoding the pore-forming $\alpha$-subunit of the cardiac voltage-dependent sodium channel have been attributed in the molecular diagnosis.4,5,10 The genetic origin can be confirmed in approximately $10-30 \%$ of patients, by the identification of mutations in the SCN5A gene. ${ }^{2,6,9}$ More than 80 mutations have been discovered, leading to many different phenotypes. ${ }^{3}$ In this report, we describe our experience in a 9-year-old boy with the BrS phenotype leading to typical ECG changes and chest pain during fever. A mutation in SCN5A gene was also discovered in the child.

\section{Case Report}

A 9-year-old boy who was previously healthy, presented to the pediatric emergency department complaining of acute chest pain accompanied by fever. He stated that he had a sore throat for 2 days with intermittent chills and fever. Then he had chest pain in 

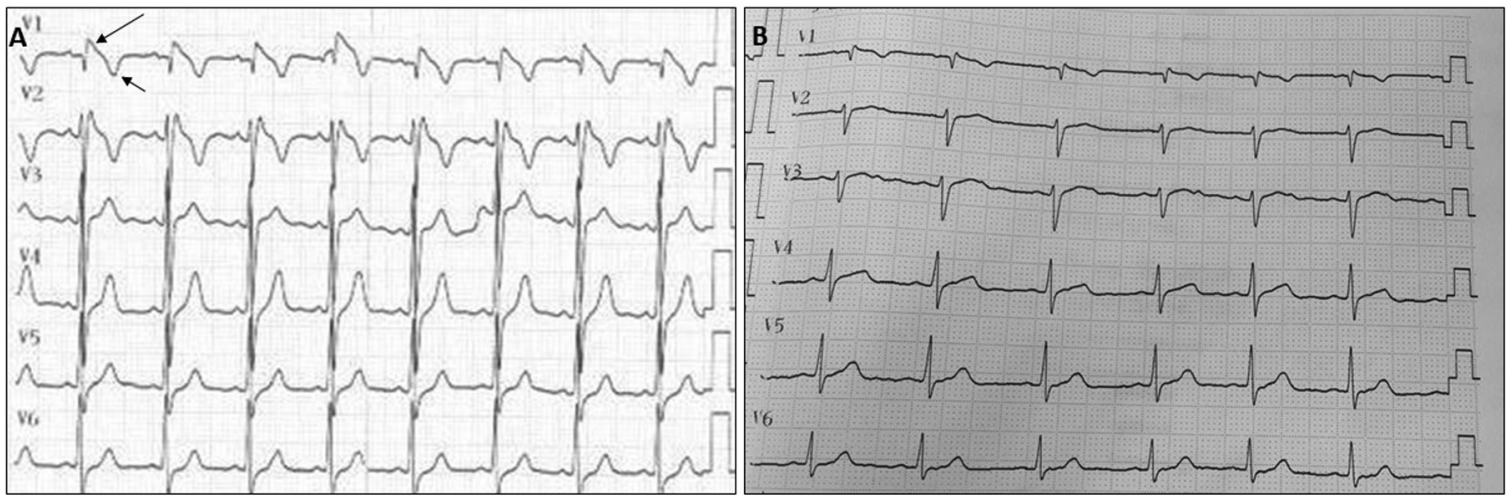

Fig. 1. ECG findings of the patient in the emergency department. V1-V6 extracted from a 12-lead ECGs. A) Coveshaped ST segment elevations larger than $2 \mathrm{~mm}$ (long arrow) in leads V1 to V2, which are followed by negative T waves (short arrow) when the patient had fever.

B) Complete resolution of the ECG changes in the ST and T waves after the fever state.

the 24 hours before admission. His pain was intermittent, compressive, non-radiating, and could not be reproduced with deep inspiration or movement. He had no nausea, vomiting, or diaphoresis. His past medical history was unremarkable with no previous history of dysrhythmia, chest pain or syncope episodes. On physical examination, he appeared well with no acute distress. He had nasal congestion and a red throat as clinical signs of an upper respiratory tract infection. His vital signs were as follows: body temperature $39.5^{\circ} \mathrm{C}$, arterial blood pressure $112 / 72 \mathrm{mmHg}$, heart rate $122 / \mathrm{min}$, respiratory rate $18 / \mathrm{min}$, and oxygen saturation $100 \%$ in room air. His cardiovascular examination was unremarkable except for a mildly rapid regular heart rate. All other examinations were also normal. Routine blood biochemistry tests, electrolytes, and cardiac enzymes (creatine kinase MB and troponin I) were within normal limits. Telecardiography was also normal. A 12-lead ECG examination revealed coved-type ST-segment elevation $>2 \mathrm{~mm}$ in the right precordial V1-V2 leads followed by negative $\mathrm{T}$ waves consistent with type $1 \mathrm{BrS}$ (Fig. 1A). After his temperature returned to normal, the chest pain ceased, and complete resolution of the changes in the ST and $\mathrm{T}$ waves were observed on an ECG (Fig. 1B). Two-dimensional echocardiography did not show any structural cardiac anomalies. The patient was then referred to another pediatric cardiology unit for further investigations. A type $1 \mathrm{BrS}$ pattern developed on the ECG after a drug challenge test with intravenous ajmaline (a class IA sodium channel blocker). The family history revealed that an uncle of the patient (II-7, Fig. 2) had died suddenly while watching a football match at the age of 25 years. The first degree family members of the patient were screened with clinical evaluation and ECG. No family members showed baseline ECG patterns suggestive of $\mathrm{BrS}$. The ajmaline challenge test was administered to family members to unmask $\mathrm{BrS}$ and it was negative for his mother (II-5), father (II-4), brother (III-1), aunt (II-6), and an uncle (II-8) (Fig. 2).

Genetic analysis was carried out on the patient and his family using peripheral venous blood. Cytogenetic analysis of the patient (III-3) revealed a 46, XYqh-karyotype with polymorphic minor deletion on the heterochromatin region of the long arm of the Y chromosome. SCN5A (NM_198056.2) gene sequence analysis on chromosome 3 p22.2 of the patient revealed homozygote c.1673A>G (rs1805124, p.H558R) point mutation at exon 11 (Applied Biosystems 3130xl Genetic Analyzer, Foster City, CA, USA) (Fig. 3). The same mutation was detected in the heterozygous state in the father (II-4), mother (II-5) and sibling (III-1) (Fig. 2).

Written informed consent was obtained from the parents for publication of this case report and any accompanying images.

\section{Discussion}

Brugada syndrome was first described by Brugada P and Brugada J in 1992 as a new cardiac electrical disease. It is characterized by syncopal episodes or SCD in affected individuals, and ECG demonstrates typical ST- 


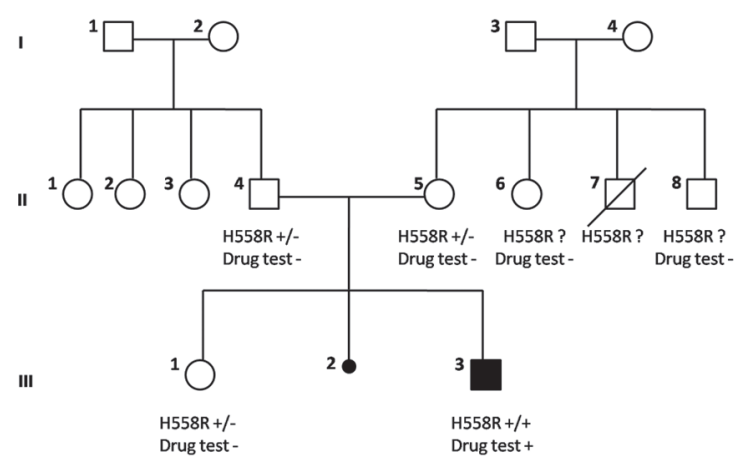

Fig. 2. Pedigree of the family with H558R mutation on SCN5A gene. The patient (III-3) was homozygous for H558R mutation, while the father (II-4), mother (II-5), and sister (III-1) was heterozygous for H558R. Drug test -: absent of typical ECG findings of the BrS when ajmaline was administered; drug test +: presence of typical ECG findings of the BrS when ajmaline was administered.

segment elevations in the right precordial leads with no structural heart disease or ischaemia. ${ }^{1}$ $\mathrm{BrS}$ is definitively diagnosed when a coved-type ST-segment elevation is observed in more than one right precordial lead in the presence or absence of a sodium channel blocking agent, and if one of the following criteria is present: (1) documented ventricular fibrillation, (2) polymorphic ventricular tachycardia, (3) a family history of SCD at $<45$ years of age, (4) coved-type ECGs in family members, (5) ventricular tachycardia can be induced with programmed electrical stimulation, syncope, or nocturnal agonal respiration. ${ }^{5}$ A cove-shaped ST elevation $\geq 2 \mathrm{~mm}$ followed by a negative $\mathrm{T}$ wave is diagnostic for type $1 \mathrm{BrS}$. The patient presented here had typical ECG findings for $\mathrm{BrS}$ and chest pain in a febrile state and had a family history of SCD at a young age. Therefore, he was diagnosed as definite type 1 BrS unmasked by fever.

The diagnosis of $\mathrm{BrS}$ can be difficult in childhood because it usually manifests as dysrhythmia or SCD, especially in young adults. ${ }^{1,3,5}$ In addition, patients with BrS may have a normal resting ECG, and BrS alterations on ECG can be unmasked by fever. It was reported that type I Brugada ECG pattern was 20 times more common in febrile patients than afebrile patients in an adult study. ${ }^{11}$ In a pediatric case series with $\mathrm{BrS}$, approximately half of the syncopal events were precipitated by fever. ${ }^{6}$ As in the case presented here, patients may not be diagnosed when fever is

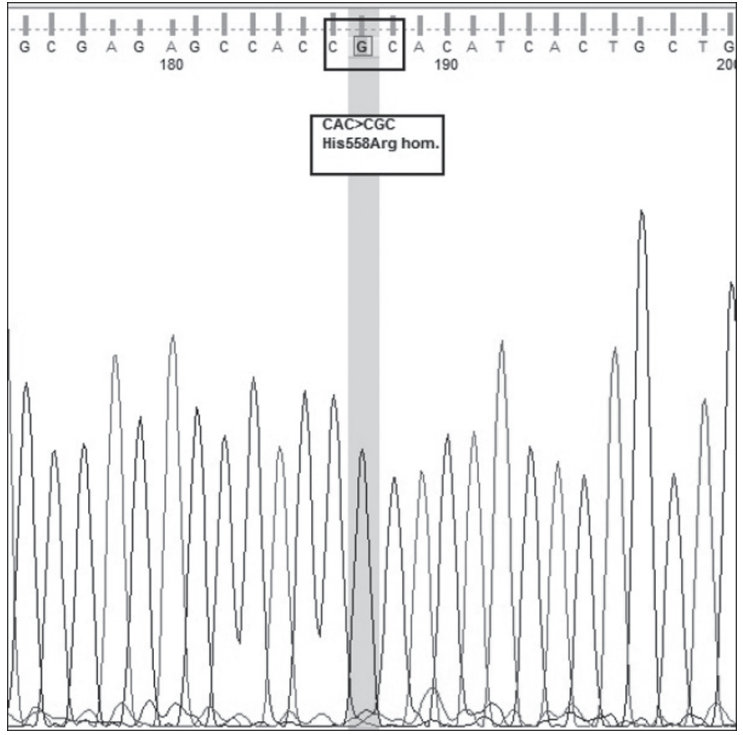

Fig. 3. Mutation identification of $S C N 5 A$ gene in the patient. Electropherogram image of $S C N 5 A$ gene sequence analysis of the proband reveals homozygous change of histidine to arginine at position 558.

not prominent and they are asymptomatic. Despite the rarity of $\mathrm{BrS}$ in children, performing an ECG seems to be rational in children with chest pain during febrile state, especially when there is a family history of SCD, as a simple but diagnostic test for BrS. Aggressive fever treatment with antipyretic agents and close follow-up are critical for a patient with BrS to prevent the induction of dysrhythmias.

Implantable cardioverter-defibrillator (ICD) placement is the only effective treatment for patients with symptomatic BrS who have survived cardiac arrest or have a history of syncope and documented ventricular dysrhythmia. However, the management of asymptomatic patients with type $1 \mathrm{BrS}$ remains controversial and risk stratification is required.5,7 It was reported that the risk of dysrhythmia was higher in previously symptomatic children and in those displaying a spontaneous ECG pattern of type I BrS. Otherwise, prognosis was favorable in asymptomatic children displaying a BrS ECG pattern only after drug challenge test. ${ }^{6}$ Therefore, ICD implantation was not considered necessary in the presented case because he was an asymptomatic young boy with normal baseline ECG having displayed a type I BrS phenotype only in a febrile state and after sodium channel blockade. Quinidine was started as a prophylactic therapy in the 
case. Although its use in children is limited with small number of patients quinidine is the only drug, which is effective in BrS, as an alternative medical treatment or as a bridge to ICD implantation. $6,7,12$

Myocardial action potentials are generated by the sequential activation and inactivation of ion channels that conduct inward $\mathrm{Na}^{+}$and $\mathrm{Ca}^{2+}$ ion currents and outward $\mathrm{K}^{+}$ion currents known as depolarization and repolarization, respectively. ${ }^{13}$ Molecular cloning of the voltagegated cardiac channels has revealed a large number of pore-forming $(\alpha)$ units responsible for the ion current. Mutations in the cardiac sodium channel gene, SCN5A, can cause a broad spectrum of inherited and potentially life-threatening arrhythmia syndromes such as dilated cardiomyopathy, long QT syndrome, and $\mathrm{BrS}$, either causing defects in the channels or on the membrane surface of cardiac cells by changing its expression. ${ }^{10,14-16}$ Most of SCN5A mutations cause an important loss-of-function by reducing the voltage-gated $\mathrm{Na}$ current available during the upstroke (phases 0) and early repolarization (phase 1) of the cardiac action potential in $\mathrm{BrS} .{ }^{4,9,13}$ Although the relationship between fever and BrS phenotype has been recognized, the molecular and cellular mechanisms underlying the fever-induced $\mathrm{BrS}$ phenomenon are not fully understood. ${ }^{4,17}$ An expression study with mutant cardiac sodium channels demonstrated a faster inactivation and slower reactivation of the sodium channel at higher temperatures, leading to further loss-of-function of sodium channel current. ${ }^{18}$ Another experimental study suggested that the temperature-dependent properties of wild-type sodium channel itself might lead to the typical $\mathrm{BrS}$ characteristics during fever. It was also demonstrated that there was no association between clinical manifestations of fever-induced BrS and any specific mutation. ${ }^{4}$

It was previously reported that $\mathrm{H} 558 \mathrm{R}$ polymorphism presents in $19 \%-24 \%$ of the human population either in the homozygote or heterozygous state. ${ }^{19,20}$ It has not been attributed as a disease-causing mutation in the Human Genome Mutation Database (HGMD). On the other hand, this common polymorphism was shown to be in the genetic background of $\mathrm{BrS}$ along with other accompanying rare mutations in the same gene by altering the biophysical effect of the mutation on the channel protein. ${ }^{20,21}$ In this case, we report a homozygote SCN5A-H558R mutation that might be responsible for the defective gating kinetics leading Brugada ECG phenotype only in cases of fever or in a drug-induced condition. The same mutation was detected in the heterozygous state in his asymptomatic father, mother, and sibling.

As a conclusion, although the diagnosis of $\mathrm{BrS}$ is rare in the pediatric population it should be considered in the differential diagnoses in patients presenting with fever and chest pain, especially when there is a family history of unexplained sudden death. Fever can trigger or exacerbate the clinical manifestations of $\mathrm{BrS}$, and should be treated aggressively due to a higher risk of ventricular dysrhythmias and SCD during febrile states. Genetic analysis should also be performed to support the clinical diagnosis in patients with a BrS-type ECG, and to identify early relatives at potential risk.

\section{Acknowledgements}

We are grateful to the patient's family for participating in this study. We thank Lorna O'Brien (www.authorserv.com) for the English language editing.

\section{REFERENCES}

1. Brugada P, Brugada J. Right bundle branch block, persistent ST segment elevation and sudden cardiac death: A distinct clinical and electrocardiographic syndrome. A multicenter report. J Am Coll Cardiol 1992; 20: 1391-1396

2. Wilde AA, Antzelevitch C, Borggrefe M, et al. Proposed diagnostic criteria for the Brugada syndrome: Consensus report. Circulation 2002; 106: 2514-2519.

3. Mivelaz Y, Di Bernardo S, Pruvot E, Meijboom EJ, Sekarski N. Brugada syndrome in childhood: A potential fatal arrhythmia not always recognised by paediatricians. A case report and review of the literature. Eur J Pediatr 2006; 165: 507-511.

4. Keller DI, Rougier JS, Kucera JP, et al. Brugada syndrome and fever: Genetic and molecular characterization of patients carrying SCN5A mutations. Cardiovasc Res 2005; 67: 510-519.

5. Antzelevitch C, Brugada P, Borggrefe M, et al. Brugada syndrome: Report of the second consensus conference: endorsed by the Heart Rhythm Society and the European Heart Rhythm Association. Circulation 2005; 111: 659-670.

6. Probst V, Denjoy I, Meregalli PG, et al. Clinical aspects and prognosis of Brugada syndrome in children. Circulation 2007; 115: 2042-2048. 
7. Vohra J. Diagnosis and management of Brugada Syndrome. Heart Lung Circ 2011; 20: 751-756.

8. De Marco S, Giannini C, Chiavaroli V, De Leonibus C, Chiarelli F, Mohn A. Brugada syndrome unmasked by febrile illness in an asymptomatic child. J Pediatr 2012; 16: 769.

9. Skinner JR, Chung SK, Nel CA, et al. Brugada syndrome masquerading as febrile seizures. Pediatrics 2007; 119: 1206-1211.

10. Chen Q, Kirsch GE, Zhang D, et al. Genetic basis and molecular mechanism for idiopathic ventricular fibrillation. Nature 1998; 392: 293-296.

11. Adler A, Topaz G, Heller K, et al. Fever-induced Brugada pattern: How common is it and what does it mean? Heart Rhythm 2013; 10:1375-1382.

12. Viskin S, Wilde AAM, Tan HL, Antzelevitch C, Shimizu W, Belhassen B. Empiric quinidine therapy for asymptomatic Brugada syndrome: Time for a prospective registry. Heart Rhythm 2009; 6: 401-404.

13. Nerbonne JM, Kass RS. Molecular physiology of cardiac repolarization. Physiol Rev 2005; 85: 1205-1213.

14. Wang Q, Shen J, Splawski I, et al. SCN5A mutations associated with an inherited cardiac arrhythmia, long QT syndrome. Cell 1995; 80: 805-811.

15. McNair WP, Ku L, Taylor MR, et al. SCN5A mutation associated with dilated cardiomyopathy, conduction disorder, and arrhythmia. Circulation 2004; 110: 21632167.
16. Ge J, Sun A, Paajanen V, et al. Molecular and clinical characterization of a novel SCN5A mutation associated with atrioventricular block and dilated cardiomyopathy. Circ Arrhythm Electrophysiol 2008; 1: 83-92.

17. Keller DI, Huang $\mathrm{H}$, Zhao J, et al. A novel SCN5A mutation, F1344S, identified in a patient with Brugada syndrome and fever-induced ventricular fibrillation. Cardiovascular Research 2006; 70: 521-529.

18. Dumaine R, Towbin JA, Brugada $\mathrm{P}$, et al. Ionic mechanisms responsible for the electrocardiographic phenotype of the Brugada syndrome are temperature dependent. Circ Res 1999; 85: 803-809.

19. Makielski JC, Ye B, Valdivia CR, et al. A ubiquitous splice variant and a common polymorphism affect heterologous expression of recombinant human SCN5A heart sodium channels. Circ Res 2003; 93: 821-828.

20. Cheng J, Morales A, Siegfried JD, et al. SCN5A rare variants in familial dilated cardiomyopathy decrease peak sodium current depending on the common polymorphism H558R and common splice variant Q1077del. Clin Transl Sci 2010; 3: 287-294.

21. Viswanathan PC, Benson DW, Balser JR. A common SCN5A polymorphism modulates the biophysical effects of an SCN5A mutation. J Clin Invest 2003; 111: 341-346. 